\title{
Electron-hole pair creation by atoms incident on a metal surface
}

\author{
J.R. Trai** and D.M. Bird \\ Department of Physics, University of Bath, Bath BA2 7AY, UK \\ M. Persson \\ Department of Applied Physics, Chalmers/Göteborg University, S-412 96 Göteborg, Sweden \\ S. Holloway \\ Surface Science Research Centre, University of Liverpool, Liverpool L69 3BX, UK
}

(Dated: March, 2003)

\begin{abstract}
Electron-hole pair creation by an adsorbate incident on a metal surface is described using $a b$ initio methods. The approach starts with standard first principles electronic structure theory, and proceeds to combine classical, quantum oscillator and time dependent density functional methods to provide a consistent description of the non-adiabatic energy transfer from adsorbate to substrate. Of particular interest is the conservation of the total energy at each level of approximation, and the importance of a spin transition as a function of the adsorbate/surface separation. Results are presented and discussed for $\mathrm{H}$ and $\mathrm{D}$ atoms incident on the $\mathrm{Cu}(111)$ surface.
\end{abstract}

PACS numbers: 73.20.Hb, 34.50.Dy, 68.43.-h

Understanding the fundamental processes involved in gas-surface interactions is an important goal, from both a pure and applied scientific perspective. In recent years much progress has been made in understanding these interactions, $\frac{1}{,}$ but a key phenomenon that has received little attention is energy dissipation into substrate degrees of freedom for an adsorbate incident upon a surface - an essentially non-adiabatic process. Energy loss by phonon excitation has recently been described successfully (notably by Wang et $a l^{\underline{2}}$ and Busnengo et al. $\frac{\underline{3}}{}$ ), but a description of the energy loss by the excitation of lowenergy electrons at metal surfaces has not been achieved for 'real' systems. This energy transfer mechanism is known to be of central importance in many situations. $\frac{1}{1}$ Here we report $a b$ initio calculations of electron-hole pair creation for $\mathrm{H} / \mathrm{Cu}(111)$.

In a previous paper $\underline{\underline{4}}$ we have described a method to calculate the classical force experienced by an atom incident on a metal surface due to excitation of electrons in the system - a nearly adiabatic process in which many low-energy electron-hole pairs are excited. This method is based upon Time Dependent Density Functional Theory (TDDFT) together with a nearly-adiabatic approximation that allows the definition of a position-dependent friction coefficient. In Ref. 4 we principally described the development of the theory and parallel algorithm necessary to obtain this friction coefficient. Preliminary results were presented for $\mathrm{H}$ on $\mathrm{Cu}(111)$, and discussed only in terms of convergence behaviour and basic viability of the method.

In a recent Letter ${ }^{\frac{5}{}}$ we have briefly described a further extension of this work. The creation of electron-hole pairs due to the adsorbate/surface interaction is described as the excitation of quantum oscillators by a driving force - the well known Forced Oscillator Model (FOM). This approach allows the prediction of the energy spectrum of excited electrons. The results obtained may be com- pared to the recent experimental work of Nienhaus and co-workers, who have directly measured the hot electrons and holes created at metal surfaces by the adsorption of thermal hydrogen and deuterium atoms in the form of a 'chemicurrent' in a Schottky diode. ${ }^{6,7,8,9}$ Good agreement was found between predicted and experimental results for $\mathrm{H} / \mathrm{Cu}(111)$.

Our aim in this paper is to provide a more complete description of the theory and approximations employed in these calculations, and to show explicitly the relationship between the classical description of the adsorbate dynamics and the quantum description of the excited electrons. A further important goal is to define clearly the approximations in the theory and their physical consequences. Of particular interest is the spin transition (from spinpolarised to non-magnetic) that can occur when an openshell adsorbate approaches a metallic surface. This spin transition is found for the $\mathrm{H} / \mathrm{Cu}$ system and in this case a direct application of the friction coefficient and FOM results in an unphysical singularity in the rate of energy transfer. In Ref. 5 we provided a brief argument as to how this problem may be resolved. Here we present a more complete discussion of this singular behaviour, together with further details of the results obtained using the FOM for the properties of the excited electronhole pairs. In a recent paper Gadzuk ${ }^{10}$ has also analysed the excitation and detection of chemicurrents. He uses a three step model consisting of (i) the excitation of hot electrons, (ii) their transport across the thin metal film of the Schottky diode, and (iii) their transmission across the metal-semiconductor interface. Our principal aim in this paper is to provide a detailed theory of step (i) that goes beyond the simple model expressions used by Gadzuk.

The approach we use may be naturally divided into three stages. A standard first principles DFT calculation of the adsorbate/surface system is carried out to obtain the Kohn-Sham (KS) states and self-consistent potential 
for the adsorbate at various positions. A friction coefficient is then obtained from the KS states and potential which gives the energy loss of the incident adsorbate due to electron-hole pair creation. This friction coefficient provides a description of the average non-adiabatic energy transfer behaviour of the system, and is derived by applying TDDFT and a nearly-adiabatic approximation. We may then solve a classical equation of motion to obtain a trajectory for the incident adsorbate. Finally the response of the electron gas to the time-varying potential due to this trajectory is found by applying the FOM to obtain a semi-classical (classical atomic motion coupled to quantum metallic electrons) description of electronhole pair creation. In the next section the formalism that leads to the friction coefficient is developed, and results given for $\mathrm{H} / \mathrm{Cu}(111)$. In section $\amalg$ the FOM is presented, together with the approximations used in our implementation, and discussed. In section III we show how this method is used to predict a variety of experimentally measurable quantities. Results for $\mathrm{H}$ and D atoms incident on the $\mathrm{Cu}(111)$ surface are presented and analysed.

\section{FRICTION COEFFICIENT}

We begin with a description of the classical motion of an adsorbate coupled to the electronic states of the substrate. An approach is required that extends beyond the Born-Oppenheimer approximation of nuclear motion on a single potential-energy surface to include non-adiabatic energy loss of the incident nuclei to the substrate electrons. The nuclei of the substrate are considered stationary, although our approach can easily be generalised to include their motion. Our goal in this section is to outline the theory used to obtain a friction description of the energy transfer (as first derived by d'Agliano et al $\stackrel{11}{\underline{13}}$ and Blandin et al $\stackrel{12}{\stackrel{1}{2} \text { and used by Hellsing and Persson } 13}$ for damping of vibrational modes; see also Head-Gordon and Tully $\underline{14}$ and Plihal and Langreth $\frac{15}{15}$ ) and apply this to the $\mathrm{H} / \mathrm{Cu}(111)$ system. Results for the friction coefficient are found to exhibit singular behaviour. We explain the source of this unphysical singularity and describe an approach taken to remove it.
The time-dependent motion of an adsorbate results in a non-adiabatic energy transfer to the many-electron system of the substrate, which can be handled in the nearly adiabatic approximation. To ease the notation we restrict the discussion to a single atomic nucleus following a trajectory $z(t)$. In general the non-adiabatic energy transfer from the nucleus to the many-electron system can be expressed as (see Appendix),

$$
\dot{E}_{\text {non-ad }}(t)=\dot{z}(t) \int d \mathbf{r} \frac{d V_{\text {ext }}(\mathbf{r}, z(t))}{d z} \delta n(\mathbf{r}, t)
$$

Here, $V_{\text {ext }}(\mathbf{r}, z)$ is the electron-nucleus interaction potential and $\delta n(\mathbf{r}, t)=n(\mathbf{r}, t)-n_{0}(\mathbf{r}, z)$ is the deviation of the instantaneous electron density $n(\mathbf{r}, t)$ from the ground state electron density $n_{0}(\mathbf{r}, z)$ when the nucleus is fixed at position $z(t)$. In our case, the time-dependent motion of the nucleus is so slow that $\delta n(\mathbf{r}, t)$ is expected to be small and can be handled in a nearly adiabatic approximation. In this approximation $\delta n(\mathbf{r}, t)$ is obtained by using the linear response of the electron system for a fixed position of the atomic nucleus to the perturbation

$$
V_{\mathrm{ext}}\left(\mathbf{r}, z\left(t^{\prime}\right)\right)-V_{\mathrm{ext}}(\mathbf{r}, z(t)) \simeq \frac{d V_{\mathrm{ext}}(\mathbf{r}, z(t))}{d z}\left(z\left(t^{\prime}\right)-z(t)\right),
$$

where in the last step we have assumed that the nucleus only moves a small distance during the electronic response time. The linear response of the many-electron system to an arbitrary perturbation $\delta V(\mathbf{r}, t)$ is given by

$$
\delta n(\mathbf{r}, t)=\int_{-\infty}^{t} d t^{\prime} \int d \mathbf{r}^{\prime} \chi\left(\mathbf{r}, \mathbf{r}^{\prime}, t-t^{\prime} ; z\right) \delta V\left(\mathbf{r}^{\prime}, t^{\prime}\right)
$$

where $\chi\left(\mathbf{r}, \mathbf{r}^{\prime}, t-t^{\prime} ; z\right)$ is the density-density response kernel for the nucleus at position $z$. Equation (3) together with the perturbation of Eq. (2) result in the following expression for the non-adiabatic energy transfer,

$$
\dot{E}_{\text {non-ad }}(t)=\dot{z}(t) \int_{-\infty}^{t} d t^{\prime} \Lambda\left(t-t^{\prime} ; z(t)\right) z\left(t^{\prime}\right)
$$

Here the memory function, $\Lambda$, is defined as

$$
\Lambda(\tau ; z)=\int d \mathbf{r} \int d \mathbf{r}^{\prime} \frac{d V_{\mathrm{ext}}(\mathbf{r}, z)}{d z}\left(\chi\left(\mathbf{r}, \mathbf{r}^{\prime}, \tau ; z\right)-\delta(\tau) \int_{0}^{\infty} d \tau^{\prime} \chi\left(\mathbf{r}, \mathbf{r}^{\prime}, \tau^{\prime} ; z\right)\right) \frac{d V_{\mathrm{ext}}\left(\mathbf{r}^{\prime}, z\right)}{d z}
$$

Note that this formalism can also be applied to vibrational damping of an adsorbate by excitation of electronhole pairs. In this case, the expansion is simply around the equilibrium position, $\underline{\underline{13}}$

The friction force description of the lossy response of the many-electron system to the adsorbate motion is now obtained by taking the slow adsorbate limit. In this case, we can use the low frequency limit of the response of the electron gas and one finds, in close analogy with the 
derivation of the vibrational damping rate, 13

$$
\Lambda(\omega ; z)=-i \eta(z) \omega+\mathcal{O}\left(\omega^{2}\right),
$$

where $\eta(z)$ is real. This low frequency approximation for $\Lambda(\omega ; z)$, inserted in Eq. (4), gives directly a friction description for the energy loss

$$
\dot{E}_{\text {non-ad }}(t)=\eta(z(t)) \dot{z}(t)^{2} .
$$

That this result gives rise to a friction force is simply understood by imposing energy conservation for the combined many-electron and nucleus system. The time derivative of the total energy then gives

$$
M \ddot{z}=-\frac{d V_{0}}{d z}-\eta(z(t)) \dot{z}
$$

where $M$ is the mass of the atomic nucleus and $V_{0}(z)$ is the ground state energy of the electronic system when the nucleus is at a fixed position $z$. In Eq. (7) it can be seen that the 'memory' of the lossy response of the electronic system is removed, hence the limit leading to Eqs. (7) and (8) can be regarded as a Markov approximation. For a complete equation of motion for the adsorbate one needs to add a stochastic force to Eq. (8) to ensure that the particle reaches thermal equilibrium. However, in our case the temperature is low and we can neglect this force.

An explicit expression for the friction coefficient can now be obtained using time-dependent density functional theory, again in close analogy with the derivation of the vibrational damping rate $\underline{\underline{13}}$ In TDDFT the response kernel at a frequency $\omega$ is approximated by the response kernel for the non-interacting KS electrons and the external field is replaced by an effective field $V_{e f f}(\mathbf{r}, \omega)$ which includes the Hartree and exchange-correlation fields. Under the widely used assumption of an adiabatic exchangecorrelation potential, the imaginary part of the frequency dependent memory function in Eq. (5) is given by $\underline{\underline{13}}$

$$
\operatorname{Im} \Lambda(\omega ; z)=-2 \pi \sum_{i, j}\left|\left\langle\psi_{i}\left|\frac{d V_{e f f}(\omega)}{d z}\right| \psi_{j}\right\rangle\right|^{2}\left(f\left(\epsilon_{i}\right)-f\left(\epsilon_{j}\right)\right) \delta\left(\hbar \omega-\epsilon_{i}+\epsilon_{j}\right)
$$

where $\psi_{i}$ and $\epsilon_{i}$ are the KS eigenstates of the electrons at position $z$ of the adsorbate, and $f(\epsilon)$ is the Fermi-Dirac occupation function. The friction coefficient is now, according to Eq. (6), obtained from the low frequency limit of $\operatorname{Im} \Lambda(\omega)$. By making a straightforward generalisation to spin-polarised TDDFT, and changing our notation slightly, we obtain $\underline{\underline{4}}$

$$
\eta(z)=\pi \hbar \sum_{\sigma} \sum_{\alpha, \alpha^{\prime}}\left|\left\langle\epsilon_{\mathrm{F}} \alpha, \sigma\left|\frac{d V_{\sigma}}{d z}\right| \epsilon_{\mathrm{F}} \alpha^{\prime}, \sigma\right\rangle\right|^{2}
$$

where $\alpha$ and $\alpha^{\prime}$ are supplementary quantum numbers for states on the Fermi surface, and $V_{\sigma}(\mathbf{r}, z)$ is the KS potential for spin $\sigma$. It is important to note that the static KS potential, $V_{\sigma}(\mathbf{r}, z)$, for spin $\sigma$ appears in Eq. (10) as a result of the low frequency limit and not due to an additional approximation or assumption, hence this is the correct result within TDDFT for an adiabatic XC functional.

The results in Eqs. (8) and (10) allow us to calculate the classical trajectory of an adsorbate interacting with a substrate, using a friction coefficient to describe the nonadiabatic energy loss to the substrate electrons. This description is valid within TDDFT (of course an approximate XC functional must be chosen for calculations) in combination with two approximations. First, we assume the substrate electrons respond nearly-adiabatically to the motion of the adsorbate in the sense that the instantaneous electron density is always close to the ground state density. The second, and most significant, approx- imation is the assumption that the adsorbate moves so slowly that the Markov limit can be taken to remove the memory of the lossy response of the electronic system. The validity of this approximation depends on the assumption of a linear frequency dependence of the memory function over the frequency range set by the timedependence of the adsorbate motion. Such a frequency dependence is expected over the range where the density of states for electron-hole pairs is essentially linear. In the case of a jellium surface the corresponding energy range is several $\mathrm{eV}$ and the Markov limit should be a good approximation for atoms with a kinetic energy of a few $\mathrm{eV} \stackrel{13}{\underline{13}}$ However, as discussed in more detail below, we find that the adsorbate motion through the spin transition is not slow, and in this case the Markov approximation fails.

\section{A. H/Cu(111): Spin transition and singularities}

We begin with a standard, self-consistent plane-wave pseudopotential calculation of the required KS states and potentials, for $\mathrm{H}$ atoms moving perpendicular to the surface above the top site of $\mathrm{Cu}(111)$. To describe the surface a five-layer slab together with a vacuum gap equivalent to another five empty layers is employed. Calculations are performed using a $2 \times 2$ in-plane super-cell, XC effects are described by a spin-polarised version of the PW91 functional, 16 a Troullier-Martins 17 pseudopotential is used for $\mathrm{Cu}$, and $\mathrm{H}$ is represented by a Coulomb potential. A plane-wave cut-off of $830 \mathrm{eV}$ is used and 54 


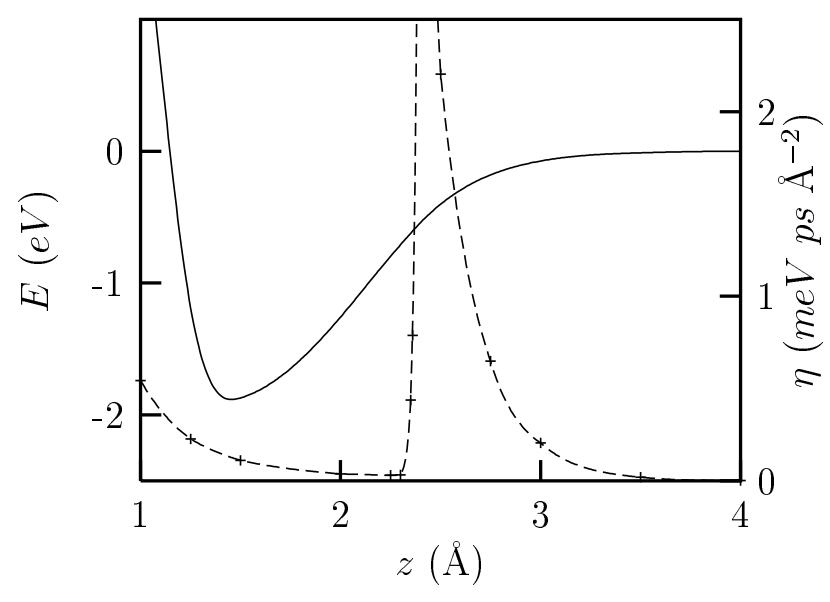

FIG. 1: Potential energy (solid line) and friction coefficient (dashed line) for $\mathrm{H}$ atom in perpendicular motion above the top site of $\mathrm{Cu}(111)$.

k-points are included in the full surface Brillouin zone, together with a Fermi surface broadening of $0.25 \mathrm{eV}$. Total energy calculations are performed for a range of heights between 1.0 and $4.0 \AA$ above the surface, and for heights $\pm h$ around these points from which the deformation potential, $d V_{\sigma} / d z$, is calculated using a finite difference. For the results given here, $h=0.02 \AA$. Full details of the calculation are given in Trail et al. Friction $^{4}$ coefficients are evaluated for the same range of heights and for motion perpendicular to the surface. Care must be taken to interpret the super-cell geometry correctly when evaluating Eq. (10) within a plane-wave basis since we are interested in the isolated motion of an atom above the surface, not the coherent motion of a mono-layer. It is also important to perform a correct discretisation of Eq. (10) within the finite available sampling of $\mathbf{k}$ space to obtain an accurate friction coefficient. $\stackrel{4}{*}$ In keeping with the natural application of spin-polarised DFT, we begin by assuming spin-adiabaticity. This means that the total energy is minimised with respect to the magnetisation density as well as the charge density. We refer to this in what follows as the 'free spin' case.

As a preliminary test it is encouraging that the friction coefficient at the total energy minimum predicts a lifetime of $0.8 \mathrm{ps}$ for the perpendicular vibrational mode of atomic hydrogen on $\mathrm{Cu}(111)$, a value that compares well with the 0.7 ps deduced from the results of Infrared Reflection Absorption Spectroscopy experiments $\frac{18}{18}$ Here we have assumed that the amplitude of the mode is small enough that no significant variation of $\eta(z)$ occurs, and that the potential is harmonic.

Figure 1] shows the friction coefficient and potential energy for the range of heights considered. It is immediately apparent that for $\mathrm{H}$ close to the surface and very far from the surface our results may be realistic, but that as the atom approaches $z_{0}=2.39 \AA$ from above the friction coefficient shows singular behaviour. Analysis of these results shows that close to $z_{0}, \eta(z) \sim\left(z-z_{0}\right)^{-1}$. We also find that $z_{0}$ is the height at which the system makes a transition from a spin-polarised $\left(z>z_{0}\right)$ to spindegenerate $\left(z \leq z_{0}\right)$ state. The changes in the electronic structure that accompany the spin transition are shown by the density of states projected onto the hydrogen $1 \mathrm{~s}$ orbital (Fig. 22). When the atom is well separated from the surface the density of states shows narrow resonances with the majority spin state fully occupied and the minority spin state empty. As the atom approaches the surface (see figure for $2.5 \AA$ ) both spin states broaden and begin to merge. At a height of $2.0 \AA$ the two spin states have become degenerate and there is no net polarisation. For closer atom-surface separations the H-related states continue to drop in energy.

The results of Fig. 1 clearly show that the singular behaviour of the friction coefficient is a consequence of the spin transition. The effect of this singularity in $\eta$ may be assessed by considering the energy loss, $\Delta E$, for an adsorbate following an arbitrary trajectory $z(t)$. For $\eta(z)=\eta_{0} z^{-1}$

$$
\begin{aligned}
\Delta E & =\int_{-\infty}^{\infty} \eta(z) v^{2} d t \\
& =\eta_{0} \int_{z_{i}}^{z_{f}} v z^{\prime}{ }^{-1} d z^{\prime},
\end{aligned}
$$

where $v=\dot{z}, z_{i}$ is the initial height and $z_{f}$ is the final height. For any real trajectory, $\Delta E$ must be finite so either the trajectory does not reach the singularity (at $z=0$ ) for any time, or at $z=0$ the velocity is zero. In other words, any real trajectory stops at or above the singularity, no matter how fast the adsorbate impinges on the surface. For example, a flat potential results in a trajectory that starts at $z_{i}$ with a velocity of $-v_{i}$, and stops at $z_{f}=z_{i} \exp \left(-\frac{m v_{i}}{\eta_{0}}\right)$. This 'infinite stopping power' is obviously unphysical, and the source of this behaviour must be identified.

We begin by showing that the singular behaviour is an expected consequence of the theory applied here, and is not a numerical artifact. Equation (10) contains the expression $d V_{\sigma} / d z$. In terms of the spin up and spin down charge densities, $\rho_{\uparrow}$ and $\rho_{\downarrow}$, we define the total charge density, $\rho=\rho_{\uparrow}+\rho_{\downarrow}$, the total spin $S=\int\left(\rho_{\uparrow}-\right.$ $\left.\rho_{\downarrow}\right) d^{3} \mathbf{r}$ and the relative distribution of magnetisation $\zeta=$ $\left(\rho_{\uparrow}-\rho_{\downarrow}\right) / S$. Expressing the charge and magnetisation densities in terms of these three quantities allows us to separate out the gross variation due to changes in total spin by giving the variation of the potential with $z$ as 

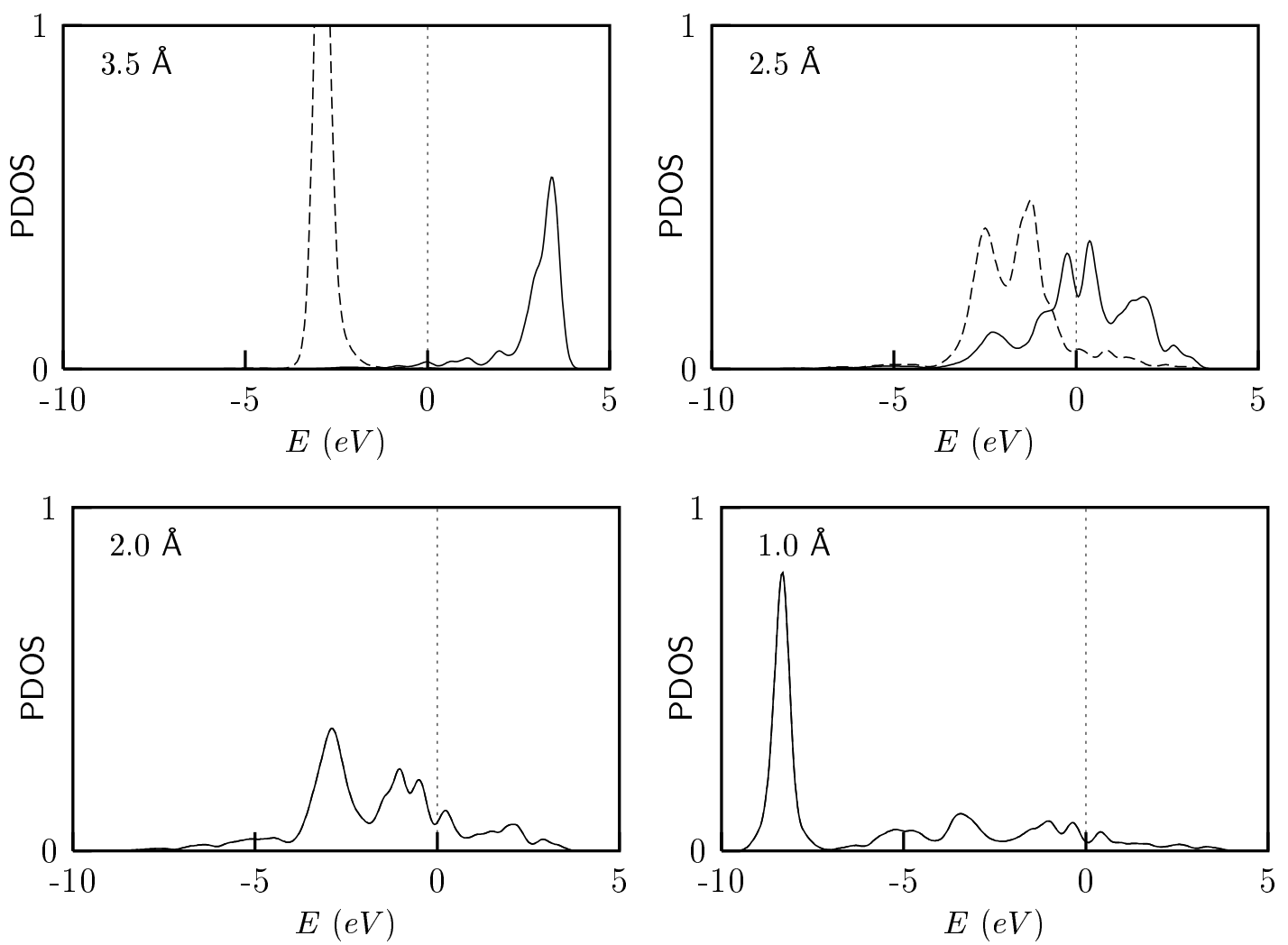

FIG. 2: Densities of states projected onto the hydrogen $1 s$ orbital at different heights above the Cu(111) surface. Dashed and solid lines show results for majority and minority spins respectively. Fermi energy is at $0 \mathrm{eV}$.

$$
\frac{d V_{\sigma}(\mathbf{r})}{d z}=\int\left(\frac{\delta V_{\sigma}(\mathbf{r})}{\delta \rho\left(\mathbf{r}^{\prime}\right)} \frac{\partial \rho\left(\mathbf{r}^{\prime}\right)}{\partial z}+\frac{\delta V_{\sigma}(\mathbf{r})}{\delta \zeta\left(\mathbf{r}^{\prime}\right)} \frac{\partial \zeta\left(\mathbf{r}^{\prime}\right)}{\partial z}\right) d^{3} \mathbf{r}^{\prime}+\frac{\partial V_{\sigma}(\mathbf{r})}{\partial S} \frac{\partial S}{\partial z}
$$

We concentrate on the third term of this equation since this explicitly describes the effect of changes in the total spin. Our calculations show that $S \sim\left(z-z_{0}\right)^{\frac{1}{2}}$, as expected for a second order phase transition within a mean field theory. This implies that, provided $\frac{\partial V_{\sigma}(\mathbf{r})}{\partial S}$ has a constant part to its variation with $S$ as $S \rightarrow 0$ (and there is no reason to believe it has not), the resulting $\left(z-z_{0}\right)^{-\frac{1}{2}}$ behaviour will dominate the deformation potential near $z_{0}$. Through the definition of the friction coefficient in Eq. (10) this results in the $\sim z^{-1}$ behaviour of $\eta$ above the transition point.

Given that the singularity arises naturally in our friction coefficient description of electron-hole pair creation, it is important to ask which of the approximations in the underlying theory breaks down in the vicinity of the spin transition. As discussed above, a major assumption is that the linear part of the expansion of $\Lambda(\omega ; z)$ in Eq. 6 provides an accurate approximation for $\operatorname{Im} \Lambda(\omega ; z)$ over the range of $\omega$ where the trajectory is significant. It is a failure of this assumption that results in the singular behaviour; any non-zero velocity of the adsorbate exactly at the spin transition means that the nearly adiabatic approximation breaks down. The spin cannot relax instantaneously, as is assumed in the free spin case. Given that some 'memory' must be retained in reality, the Markov approximation cannot provide a realistic description close to the spin transition. The timescale for spin relaxation will in practice be set by the rate of hopping of electrons between adsorbate and substrate and this in turn is governed by the width of the projected density of states shown in Fig. 2. How can the unphysically fast spin relaxation that naturally arises in our nearly adiabatic theory be prevented? We have chosen to adopt the simplest solution to this problem, namely to keep the total spin fixed for the whole trajectory of the adsorbate. Our justification for this derives largely from the results 


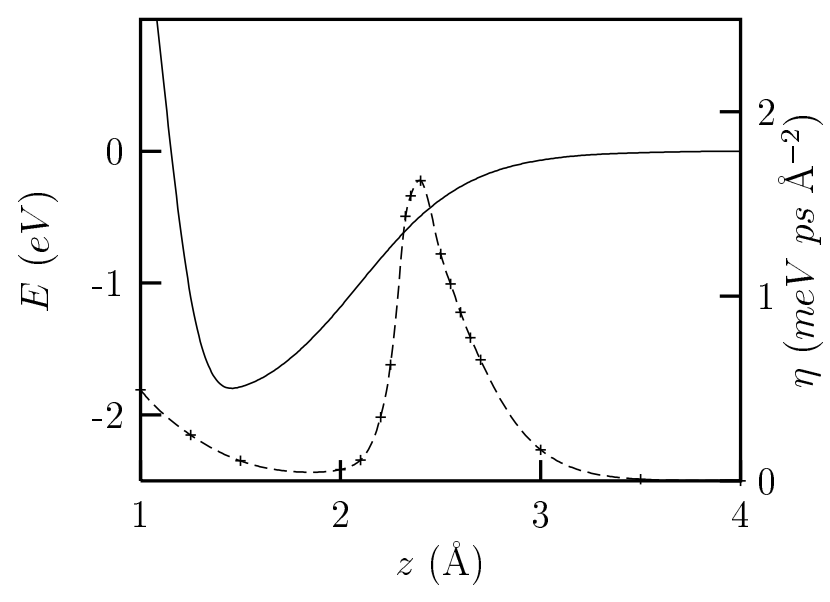

FIG. 3: Potential energy (solid line) and friction coefficient (dashed line) for $\mathrm{H}$ atom in perpendicular motion above the top site of $\mathrm{Cu}(111)$. Results are obtained using the fixed spin approximation.

we obtain. We show below that, except in the immediate vicinity of the transition point, the potential energy and friction coefficient are very similar for the free spin and fixed spin cases. Also, it is shown in section III that the peak in the friction coefficient near $z_{0}$ does not provide a dominant contribution either to the total rate of electronhole pair creation or to the spectrum of the electron hole pairs, provided the singularity is smoothed. Essentially, our results show that, provided the unphysical singularity is removed in a reasonable way, the results for electronhole pair creation are not strongly dependent on the way in which the singularity is smoothed. In another paper we address this point in more detail, by using the NewnsAnderson model of the adsorbate-substrate interaction to analyse the dynamics close to the spin transition. $\underline{19}$

For the free spin case Eq. (12) can be viewed as $\lim _{\omega \rightarrow 0}$ of the derivative of the TDDFT effective potential, as required in Eq. (9). The fixed spin approximation takes $\lim _{\omega \rightarrow 0}$ for the variation of the charge and spin densities, but $\lim _{\omega \rightarrow \infty}$ for the variation of the total spin, $S$. Essentially this corresponds to assuming that the total charge density, $\rho$, and the relative distribution of magnetisation, $\zeta$, respond adiabatically to the motion of the incident adsorbate, but the total spin, $S$, responds in the opposite impulsive limit - that is, for the time scales considered, the total spin does not have time to change. For the fixed spin case Eq. (12) is replaced by

$$
\left.\frac{d V_{\sigma}(\mathbf{r})}{d z}\right|_{S}=\int \frac{\delta V_{\sigma}(\mathbf{r})}{\delta \rho\left(\mathbf{r}^{\prime}\right)} \frac{\partial \rho\left(\mathbf{r}^{\prime}\right)}{\partial z}+\left.\frac{\delta V_{\sigma}(\mathbf{r})}{\delta m\left(\mathbf{r}^{\prime}\right)} \frac{\partial m\left(\mathbf{r}^{\prime}\right)}{\partial z}\right|_{S} d^{3} \mathbf{r}^{\prime},
$$

where $m(\mathbf{r})=\rho_{\uparrow}-\rho_{\downarrow}$ is the standard KS magnetisation density, and variations are constrained such that $S$ is unchanged $\stackrel{20}{=}$ In this form the $z^{-1}$ singularity due to the change in total spin is removed.
Figure 3 shows the friction coefficient and potential energy for the fixed spin approximation with $S=1$, the total spin for the $\mathrm{H}$ atom far from the surface. The potential energy agrees well with the previous free spin results - the maximum difference occurs at the minimum, where the fixed spin energy is $\sim 0.08 \mathrm{eV}$ greater than that for free spin. Agreement between the calculated $\eta(z)$ away from the transition height $\left(z_{0}=2.39 \AA\right)$ is also good. Since no singularity is present the problem of an infinite stopping power is removed.

\section{FORCED OSCILLATOR MODEL}

In the previous section a semi-classical description of the motion of an adsorbate was given, in the sense that the classical motion of the adsorbate atom can be obtained by taking into account the energy losses due to excitations of the electrons of the metal surface. In this section our aim is to describe in more detail the excitation of the electron gas itself. This will enable us to obtain the spectrum of the excited electron-hole pairs and thus make a connection to a variety of experimentally measurable quantities. The basis of the approach is the well known Forced Oscillator Model (FOM), where here the oscillators are electron-hole pairs, and these are 'forced' by the changing potential due to the motion of the adsorbate. Past applications of the FOM have involved the investigation of simple model systems for electron-hole pairs (see, for example, Schönhammer and Gunnarson 21,22,23,24 , Minnhagen 25 and Brako and Newns ${ }^{26}$ ) and more detailed phonon models (Persson and Harris 27.28$)$. Our goal here is to describe a FOM approach rooted in our ab initio description of friction, and assess to what extent the FOM is consistent with TDDFT.

We begin with the energy distribution function, $P(\omega)$, defined as the probability that the electron gas, subjected to a potential $V(t)$, is excited to energy $\hbar \omega$ above the ground state, after the interaction has taken place $\stackrel{21}{2 y}$ applying a second order cumulant expansion ${ }^{29}$ to the appropriate matrix element it is possible to obtain an approximation to $P(\omega)$ that is exact for some special cases, and is a good approximation for slowly varying potentials of arbitrary magnitude. Within this truncated cumulant expansion $P(\omega)$ is given by 21

$$
P(\omega)=\frac{1}{2 \pi} \int_{-\infty}^{\infty} \mathrm{e}^{P_{s}(t)-\alpha_{0}} \mathrm{e}^{\mathrm{i} \omega t} d t
$$

where $\alpha_{0}=\int_{0}^{\infty} P_{s}(\omega) d \omega$ is the average number of electron-hole pairs excited, $\mathrm{e}^{-\alpha_{0}}$ is the Debye-Waller factor and gives the probability of the system remaining unexcited, and

$$
P_{s}(t)=\int_{0}^{\infty} P_{s}(\omega) \mathrm{e}^{-\mathrm{i} \omega t} d \omega .
$$

Equations (14) and (15) may be understood as a multiexcitation expansion of the probability of the electron 
gas being excited by $\hbar \omega$ after infinite time has passed, in terms of the probability that $n$ electron-hole excitation events have occurred, and the probability that $n$ electron-hole excitation events will excite the electron gas by energy $\hbar \omega$. Here, $P_{s}(\omega) / \alpha_{0}$ can be interpreted as the probability that a single electron-hole excitation event is of energy $\hbar \omega$.

For non-interacting electrons, and $\omega>0$ (defining $P_{s}(\omega)=0$ for $\left.\omega<0\right), P_{s}(\omega)$ is given by ${ }^{21}$

$$
P_{s}(\omega)=\frac{1}{\hbar^{2} \omega^{2}} \sum_{i j}\left|\int_{-\infty}^{\infty}\left\langle\psi_{i}(t)|\dot{V}| \psi_{j}(t)\right\rangle d t\right|^{2} \delta\left(\omega-\omega_{i}+\omega_{j}\right)\left(f\left(\omega_{i}\right)-f\left(\omega_{j}\right)\right)
$$

where $\left|\psi_{i}(t)\right\rangle$ are the time dependent one-electron states that are initially eigenstates of the unperturbed Hamiltonian, with eigenvalues $\omega_{i}$, and evolve via the full perturbed Hamiltonian. $\dot{V}(t)$ is the time derivative of the perturbing potential. As described by Schönhammer and Gunnarsson $^{21}$ this expression may be simplified by making two assumptions. First we assume that $V(t)$ is slowly varying, so the $\left|\psi_{i}(t)\right\rangle$ are well approximated by the time-dependent adiabatic states of the full Hamiltonian, $\mathrm{e}^{-\mathrm{i} \omega_{i} t}\left|\psi_{i}\right\rangle_{t}$. Physically, this adiabatic approximation implies that the probability of electron-hole excitation is independent of the number of excitation events that have occurred. For a slow variation of $V(t)$ on a time scale $T$, only states for which $\left|\hbar \omega_{i}-\epsilon_{F}\right| \ll \hbar / T$ contribute to Eq. (16). This suggests that the matrix elements may be well described by the values taken at the Fermi level, the second approximation by Schönhammer and Gunnarsson. With these assumptions, Eq. (16) becomes

$$
P_{s}(\omega)=\frac{1}{\omega} \sum_{\alpha, \alpha^{\prime}, \sigma}\left|\int_{-\infty}^{\infty}\left\langle\epsilon_{\mathrm{F}} \alpha, \sigma|\dot{V}| \epsilon_{\mathrm{F}} \alpha^{\prime}, \sigma\right\rangle \mathrm{e}^{-\mathrm{i} \omega t} \mathrm{~d} t\right|^{2}
$$

where $\left|\epsilon_{\mathrm{F}} \alpha, \sigma\right\rangle$ denotes a stationary single particle state of spin $\sigma$ on the Fermi surface, and a supplementary quantum number, $\alpha$, has been introduced (as in Eq. (10)).

So far we have not stated what the non-interacting electron system actually is. We now show that, if KohnSham states and the KS potential are used in Eq. (17), then the rate of energy gain by the electron gas given by Eqs. (14) and (17) is identical to the energy loss rate of the adsorbate due to friction, given by Eqs. (7) and (10). It is shown in the Appendix (see also Ref ${ }^{25}$ ) that, within the FOM, the rate at which the electrons gain energy by the non-adiabatic process is

$$
\dot{E}_{\text {non-ad }}(t)=\hbar \int_{0}^{\infty} \omega \frac{\partial}{\partial t} P_{s}(\omega, t) d \omega
$$

where the $t$ variable in $P_{s}(\omega, t)$ has been introduced as a new upper limit to the integral in Eq. (16) or (17). Substitution of Eq. (17) into Eq. (18) and integration over $\omega$ then gives

$$
\dot{E}_{\text {non-ad }}(t)=\pi \hbar \sum_{\alpha, \alpha^{\prime}, \sigma}\left|\left\langle\epsilon_{\mathrm{F}} \alpha, \sigma|\dot{V}| \epsilon_{\mathrm{F}} \alpha^{\prime}, \sigma\right\rangle\right|^{2}=\eta(z) \dot{z}^{2}
$$

This shows that the FOM implemented with adiabatic KS states and matrix elements taken at the Fermi energy gives the same average energy gain as the TDDFT description within the Markov approximation.

The close connection between the TDDFT and FOM approaches to energy transfer from adsorbate to substrate implies that the FOM can be used to provide a theory of the excitation spectrum of the electron gas (that is, $P(\omega)$ and $P_{s}(\omega)$ ) that is consistent with the friction description presented in the previous section. This connection between TDDFT and the FOM means that the singularity associated with the spin transition will also be present in the FOM. This is clear in Eq. (17) where $\dot{V}$ is the derivative of the Kohn-Sham potential. For the free spin case $\dot{V}$ will be singular at the spin transition, resulting in unphysical behaviour. The argument that led to constraining the total spin to be constant, thus removing the singular behaviour, is therefore as relevant to the FOM as to the friction description of the energy transfer.

Equation (17) may be evaluated by the same approach used to obtain the friction coefficient, however this requires the storage and interpolation of a large number of matrix elements. Due to the approximations already made to develop the theory to this point this effort does not seem justifiable. Instead, we adopt an analogue of models used by past authors to describe Fermion excitations of this nature $1,21,25,26,30$ It is well established that if the eigenstates can be chosen such that the matrix element $\left\langle\epsilon_{\mathrm{F}} \alpha, \sigma|\dot{V}| \epsilon_{\mathrm{F}} \alpha^{\prime}, \sigma\right\rangle$ that appears in Eq. (17) is diagonal in $\alpha, \alpha^{\prime}$ then the system corresponds to the excitation of Tomonaga bosons that describe the electronhole pairs ${ }^{21}$. Here we seek a useful approximation to Eq. (17) that describes the excitation of a system of 'average' bosons, the properties of which vary with $z$. This is achieved by assuming that the dependence of the matrix elements in Eq. (17) on $z$ and $\alpha, \alpha^{\prime}$ can be expressed in the separable form $f_{\alpha, \alpha^{\prime}} g(z)$. Physically, this implies that each electron-hole pair excitation expe- 
riences a time-dependent 'force', of the same functional form, but with different strengths. Introducing this factorisation leads to

$$
P_{s}(\omega)=\sum_{\sigma} \frac{1}{\pi \hbar \omega}\left|\int_{-\infty}^{\infty} \eta_{\sigma}^{\frac{1}{2}}(z) \dot{z}(t) \mathrm{e}^{-\mathrm{i} \omega t} \mathrm{~d} t\right|^{2}
$$

where different spin terms are shown explicitly (subscript $\sigma$ ) and $\eta_{\sigma}$ is given by Eq. (10) with no sum over spin. Equation (20) is the final expression used to define the FOM for the calculations reported here.

Although there is no rigorous justification for this simplified form of $P_{s}(\omega)$, there are several reasons for expecting it to provide a useful description of the statistics of the energy loss. Like Eq. (17), Eq. (20) gives exactly the same average rate of energy transfer between adsorbate and electron gas as the classical friction coefficient. A similar 'single channel' approach has been used by previous authors $1,24,26,28$ in constructing model descriptions of energy loss processes at surfaces using the FOM, and this approximation is also exact for other special cases, such as for symmetry reasons or if the excited electrons are predominately $s$ in character 21 . In order to implement the FOM in this form only the friction coefficient and trajectory of the incident particle are required. From these, the probability distribution function for single electronhole pair excitation, $P_{s}(\omega)$, may be calculated and this function, through Eq. (14), defines completely the excitation of the electron gas.

\section{RESULTS}

In this section we present results for the $\mathrm{H} / \mathrm{Cu}(111)$ adsorbate/surface system. A standard Verlet integration of Eq. (8) is performed with the fixed-spin friction coefficient shown in Fig. 3 to obtain the classical trajectory of an incident $\mathrm{H}$ atom, for a range of initial kinetic energies. The resulting trajectories show expected features with a critical initial kinetic energy $\left(\epsilon_{c}\right)$ above which the atom escapes from the well, and below which it is trapped. For $\mathrm{H} / \mathrm{Cu}(111), \epsilon_{c}=0.166 \mathrm{eV}$, and for an atom that escapes from the well we find the round-trip time (for which $z(t)<3.0 \AA$ ) is $\sim 0.04$ ps.

The next step is to apply Eqs. (14), (15) and (20) to implement the FOM and extract physically measurable quantities from it. A characteristic property of the adsorbate/surface interaction is the sticking coefficient, $S(\omega)$, defined as the probability that an incident adsorbate loses sufficient energy to be captured by the surface. Here we consider only the contribution to sticking due to the energy loss via electron-hole pair production. In terms of the probability for energy gain by the substrate defined in (14) the sticking coefficient becomes

$$
S\left(\omega_{i}\right)=\int_{\omega_{i}}^{\infty} P(\omega) d \omega
$$

where $\hbar \omega_{i}=\epsilon_{i}$ is the initial kinetic energy of the adsorbate. Following the approach described by Schönhammer

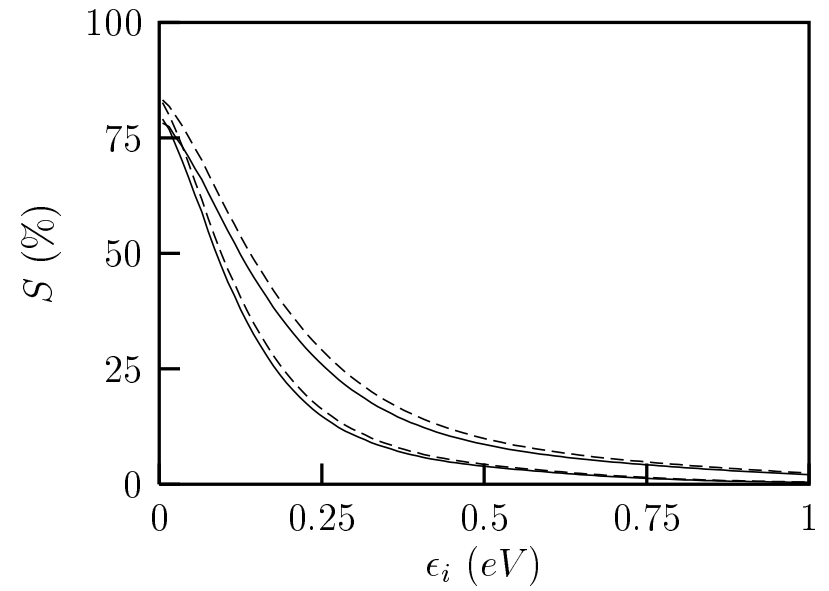

FIG. 4: Sticking coefficients for $\mathrm{H}$ and $\mathrm{D}$ atoms perpendicularly incident on the top site of $\mathrm{Cu}(111)$. Elastic trajectory is dashed line, inelastic trajectory is solid line. Upper and lower curves are for $\mathrm{H}$ and $\mathrm{D}$ respectively.

and Gunnarsson 21 a trajectory is chosen that travels into the surface and out, but is truncated at the next turning point if there is one. The use of this trajectory results in a $P(\omega)$ that is the probability that the electron gas is excited by an energy $\hbar \omega$ in the time taken for the adsorbate to cross the surface-adsorbate well twice. If this energy loss is larger than the initial kinetic energy of the adsorbate then it is considered to be captured. Past applications of the FOM to this type of problem have generally employed simple model Hamiltonians and elastic trajectories, hence do not take into account the influence of the loss of energy on the trajectory itself. Here calculations have been performed for both the elastic and truncated inelastic trajectories.

Figure 4 shows the sticking coefficient as a function of the incident kinetic energy. As expected there is an overestimate of $S\left(\omega_{i}\right)$ for the elastic trajectory due to the increased velocity. Generally, $S\left(\omega_{i}\right)$ falls from $\sim 80 \%$ at $\epsilon_{i}=0$ to $\sim 40 \%$ at the classical critical initial kinetic energy, $\epsilon_{c}$, and then falls smoothly to zero. It is important to note that this sticking coefficient does not take into account any energy loss due to phonon excitations and so it may not be compared directly to experiment. This is immediately apparent if we consider $\mathrm{H}$ incident on $\mathrm{Cu}$ at $300 \mathrm{~K}$. Experimental results and previous theoretical estimates 31 suggest $S \sim 1$, whereas for $\epsilon_{i}=\frac{3}{2} k T=38.8$ $\mathrm{meV}(T=300 \mathrm{~K}$, the results vary slowly with incident energy in the thermal range, and taking a Boltzmann average makes no discernible difference) our calculation gives $S=0.68-0.72$.

A quantity of interest is the number of electron-hole pairs excited in the process of a single $\mathrm{H}$ atom being captured by the surface. The probability that $n$ electronhole pairs are excited by the adsorbate surface interaction 
is given by the Poisson distribution

$$
P_{n}=\mathrm{e}^{-\alpha_{0}} \alpha_{0}^{n} / n !
$$

where $\alpha_{0}$ is the average number of electron-hole pairs excited, as defined earlier. Figure 5 shows $P_{n}$ for $\mathrm{H} / \mathrm{Cu}(111)$ for an inelastic trajectory that follows the $\mathrm{H}$ atom all the way to the bottom of the potential well. $\alpha_{0}$ takes the value of 11.4 electrons which, combined with a total excitation of $\sim 1.8 \mathrm{eV}$, implies that on average each excitation is of order $0.15 \mathrm{eV}$. This supports the premise that underlies our approach to calculating the energy transfer from adsorbate to substrate; namely that the process is nearly adiabatic and involves the excitation of multiple, low-energy electron-hole pairs.

\section{A. Chemicurrents}

Of particular interest here, and not investigated before using $a b$ initio methods, is the creation of hot electrons and holes during the adsorption process. In a number of papers Nienhaus et al. describe experimental investigations of the electronic excitation behaviour for a variety of adsorbates and metal surfaces $6,7,8,9$. They construct a Schottky diode device consisting of a clean metal film deposited onto a $\mathrm{Si}$ wafer. Incident atoms or molecules may excite hot electrons (or holes) at this metal surface and electrons with energies high enough to surmount the Schottky barrier can be detected as a current, referred to as a 'chemicurrent'.

The electrons detected in these experiments can be thought of as undergoing two processes. First they are excited to sufficient energy to be detected and second a number of geometrical factors and scattering mechanisms within the detector cause signal loss. The second of these has been discussed in detail by Nienhaus $, \frac{6}{7}, 8,9$

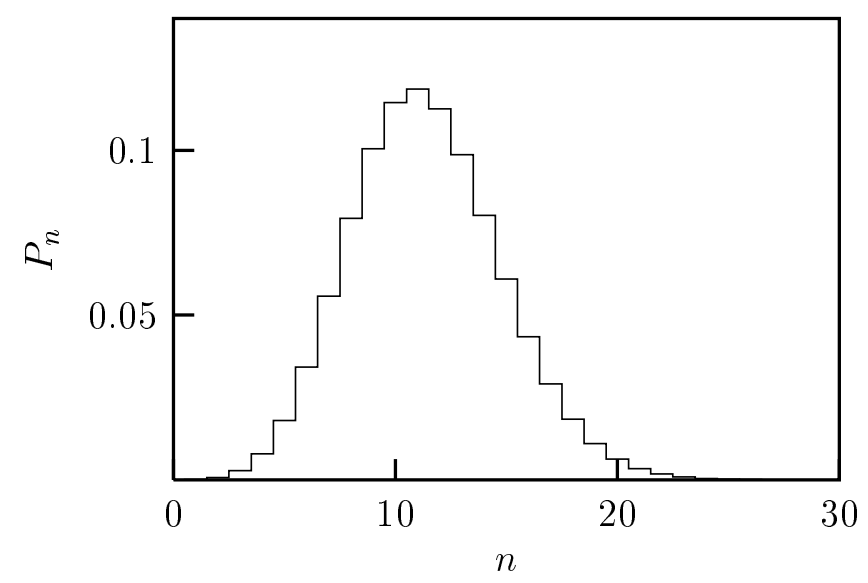

FIG. 5: Probability of excitation process creating $n$ electronhole pairs, $P_{n}$.

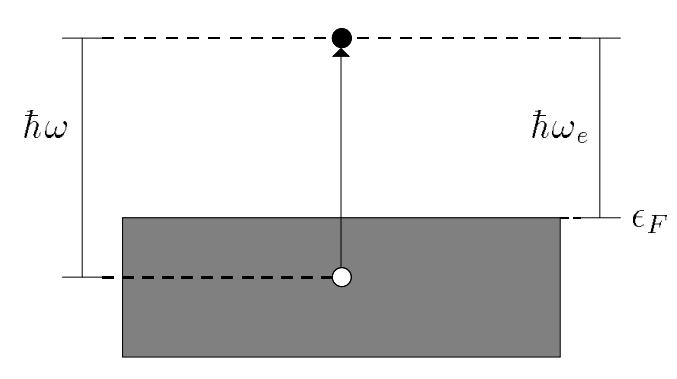

FIG. 6: An electron is excited from below the Fermi energy by $\hbar \omega$ to a state $\hbar \omega_{e}$ above the Fermi energy. The probability for this excitation to occur depends only on the electron-hole pair energy, $\hbar \omega$, provided $0<\omega_{e}<\omega$. For $\omega_{e}>\omega$ and $\omega_{e}<0$ this probability is zero.

and Gadzuk 10 , and a simple model to describe these processes results in good agreement between our results and experiment, as discussed in Ref. 5. Here we do not consider device losses, instead we concentrate on the fundamental quantity of the number of electrons excited above a specific energy. We refer to this quantity as 'electrons made available for detection'.

The number of electrons made available for detection over a Schottky barrier of $\hbar \omega_{s}$ by the adsorbate/surface interaction is written as $N_{e}\left(\omega>\omega_{s}\right)$. To obtain $N_{e}$ we must transform from excitation statistics in terms of the energy of electron-hole pairs to statistics in terms of electron energies. The probability that a single excitation event results in an electron-hole pair of energy $\hbar \omega$, with the electron possessing an energy of $\hbar \omega_{e}$, can be written as

$$
\frac{P_{s}\left(\omega, \omega_{e}\right)}{\alpha_{0}}=\frac{1}{\alpha_{0}} \frac{P_{s}(\omega)}{\omega} \Theta\left(\omega-\omega_{e}\right) \Theta\left(\omega_{e}\right) .
$$

Here $P_{s}(\omega) / \alpha_{0}$ is the probability that a single excitation event results in an electron-hole pair of energy $\hbar \omega$, as defined by Eq. (20), and $\Theta(x)=0,1$ for $x<0, x \geq 0$. Equation (23) is obtained by noting that an electron-hole pair of energy $\hbar \omega$ will consist of electrons distributed over the energy range $0<\hbar \omega_{e}<\hbar \omega$ with equal probability (see Fig. 6 ). The probability that a single excitation event results in an electron of energy $\hbar \omega_{e}$ may then be obtained by integrating Eq. (23) over all electron-hole energies, resulting in

$$
\frac{P_{e}\left(\omega_{e}\right)}{\alpha_{0}}=\frac{1}{\alpha_{0}} \int_{\omega_{e}}^{\infty} \frac{P_{s}(\omega)}{\omega} d \omega .
$$

The total number density of electrons excited to energy $\hbar \omega$ by all events in a multi-excitation expansion is then $P_{e}(\omega)$. It follows that the number of electrons made available for detection can be expressed as

$$
N_{e}\left(\omega>\omega_{s}\right)=\int_{\omega_{s}}^{\infty} \mathrm{d} \omega \int_{\omega}^{\infty} \mathrm{d} \omega^{\prime} \frac{P_{s}\left(\omega^{\prime}\right)}{\omega^{\prime}} .
$$




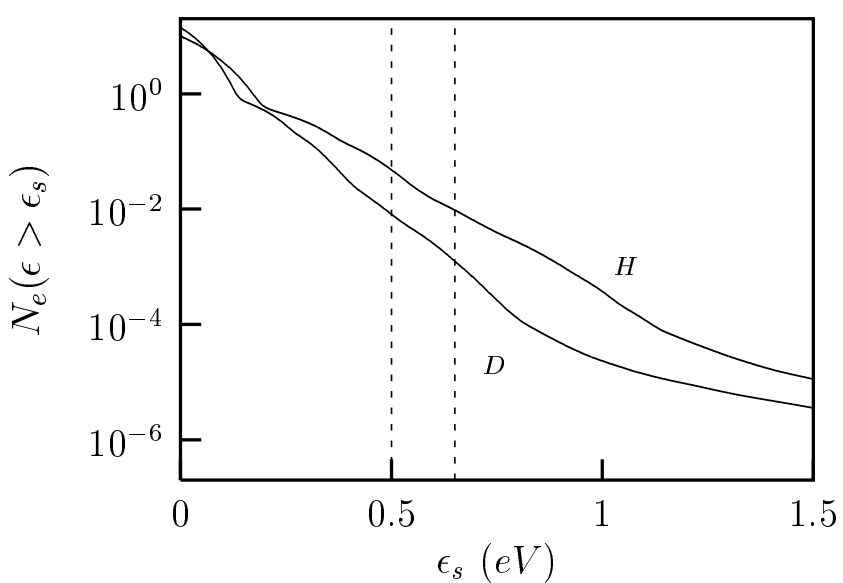

FIG. 7: Number of electrons per atom made available for detection over a Schottky barrier of height $\epsilon_{s}$ for $\mathrm{H}$ and $\mathrm{D}$ incident on the top site of $\mathrm{Cu}(111)$. The vertical lines span the range of Schottky barrier heights found by Nienhaus et $a l . \underline{6}$ for both $\mathrm{Cu}$ and $\mathrm{Ag}$.

Figure 7 shows $N_{e}\left(\omega>\omega_{s}\right)$ for a range of barrier heights, and for $\mathrm{H}$ and $\mathrm{D}$ normally incident on the top site of $\mathrm{Cu}(111)$. As in Fig. 团 the incident energy is $38.8 \mathrm{meV}$, and full inelastic trajectories are used. The data shows an essentially exponential behaviour over most of the range considered, and most of the difference between the results for $\mathrm{H}$ and $\mathrm{D}$ may be accounted for by scaling $\epsilon_{s}$ by $\sqrt{2}$ (the agreement would be exact for an elastic trajectory). This dynamic scaling is a consequence of Eq. (20) and the scaling of velocity with mass for a given kinetic energy. The unusually strong isotope effect apparent in Fig. 7 is consistent with the experimentally measured chemicurrent. Nienhaus et al $\underline{\underline{6}}$ reported no results for D incident on $\mathrm{Cu}$, but for $\mathrm{Ag}$ the detected number of electrons per atom for $\mathrm{H}$ was larger than for $\mathrm{D}$ by a factor of $\sim 6$. Figure 8 shows the calculated ratio of chemicurrents for $\mathrm{H}$ and $\mathrm{D}$, and in the range of relevant barrier heights we find an isotope effect close to the experimental result. This comparison is particularly useful as it is not necessary to consider device losses if we take the signal to be suppressed by the same factor for both $\mathrm{H}$ and $\mathrm{D}$ incident upon the detector.

It is interesting to ask where in the trajectory most of the relatively high-energy electrons that are detected as a chemicurrent are produced. This is significant because of the discussion in Section I concerning our method for removing the singularity in the friction coefficient caused by the spin transition. By keeping the spin fixed the singularity is removed, but the friction coefficient is still peaked at $z_{0}=2.39 \AA$ A. Given the rather ad-hoc way in which this peak is derived, if we find that it makes a major contribution to the predicted chemicurrent then the basis of our results could be questioned. If, however, the chemicurrent is relatively insensitive to the peak in $\eta$,

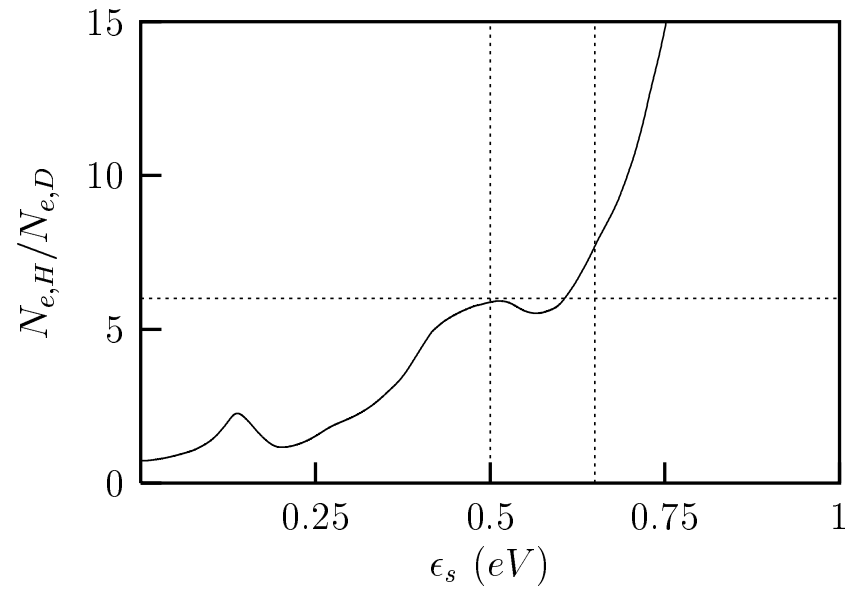

FIG. 8: The predicted ratio of the chemicurrents due to $\mathrm{H}$ and $\mathrm{D}$ incident on the top site of $\mathrm{Cu}(111)$ as a function of Schottky barrier height. The vertical lines span the range of Schottky barrier heights found by Nienhaus et al. ${ }^{6}$ for both $\mathrm{Cu}$ and $\mathrm{Ag}$. The horizontal line is the ratio of chemicurrents found experimentally for $\mathrm{Ag}\left(\epsilon_{s}=0.5-0.55 \mathrm{eV}\right)$.

then we can have more confidence in our results. Equation (20) shows that the spectrum of electron-hole pairs is determined by two factors, the speed of the adsorbate, $\dot{z}$, and the friction coefficient, $\eta$. These peak in very different places in the trajectory; $\dot{z}$ is greatest at the minimum of the potential well (at about $1.4 \AA$ ) while $\eta$ peaks at $z_{0}=2.39 \AA$. Which dominates the chemicurrent?

To address this question we (arbitrarily) separate the friction coefficient into two parts, a 'smooth' part

$$
\eta_{\sigma}^{s m}(z)= \begin{cases}\eta_{\sigma}(z) & z \leq 1.85 \AA \\ \eta_{\sigma}(1.85) \mathrm{e}^{-2(z-1.85)^{2}} & z>1.85 \AA\end{cases}
$$

and a 'peak' part that is zero for $z \leq 1.85 \AA$,

$$
\eta_{\sigma}^{p k}(z)=\eta_{\sigma}(z)-\eta_{\sigma}^{s m}(z) .
$$

The value $z=1.85 \AA$ is chosen to be the minimum of $\eta$ between $z=1$ and $2 \AA$ (see Fig. 3), and the function introduced in Eq. (26) is chosen to decay smoothly from $z=1.85 \AA$ to a value below the total friction at $z=4 \AA$. This continuous decay prevents a discontinuity in the integrand of Eq. (20) which would result in spurious high energy electron-hole pair excitations. Calculations are carried out as before, using the full friction coefficient to obtain the trajectories, but using either $\eta_{\sigma}^{s m}$ or $\eta_{\sigma}^{p k}$ to evaluate measurable quantities from the FOM. This allows us to analyse the statistics of electron-hole pair creation due to each part of the friction coefficient separately. For example, we find that $\sim 25 \%$ of the total energy transfered from the incident atom to electron-hole pairs is due to the peak, indicating that this region is significant, but not of primary importance. Figure 9 shows 
$N_{e}\left(\epsilon>\epsilon_{s}\right)$ obtained from the smooth and peaked parts, in comparison to that obtained from the complete friction profile. It should be stressed that the sum of the first two quantities is not equal to the last, due to the factor of $\sqrt{\eta}$ in Eq. (20). It can be seen that the presence or absence of the peaked region of $\eta$ makes little difference to the predicted number of electrons made available for detection, at least for the Schottky barrier heights considered here. We conclude that the interaction of the incident atom with the electron-hole pairs of the substrate is not dominated by the peak found in the calculated friction coefficient. This suggests that, provided the peak does not become singular, its precise form will not have a great bearing on our predictions of experimental observables.

Finally, we have not so far addressed an important question: what influence would the introduction of energy loss via the creation of phonons have on our estimates for the detected chemicurrent? A full answer to this would require a molecular dynamics description of the interaction, including electron-hole pair creation, and is beyond currently available computing resources. However, Strömquist et $a l^{31}$ have performed molecular dynamics calculations for $\mathrm{H} / \mathrm{Cu}(111)$ (not including electron-hole pair creation) using a model energy surface fitted to $a b$ initio data, and sampling a large number of trajectories. They obtain an energy relaxation rate of $\sim 0.7 \mathrm{ps}^{-1}$ due to phonon creation, a value that is similar to the rate of energy loss due to electron-hole pairs for the perpendicular vibrational mode of $\mathrm{H}$ on $\mathrm{Cu}(111)$ discussed above. We may obtain a crude estimate for the influence of phonon creation on our results by intro-

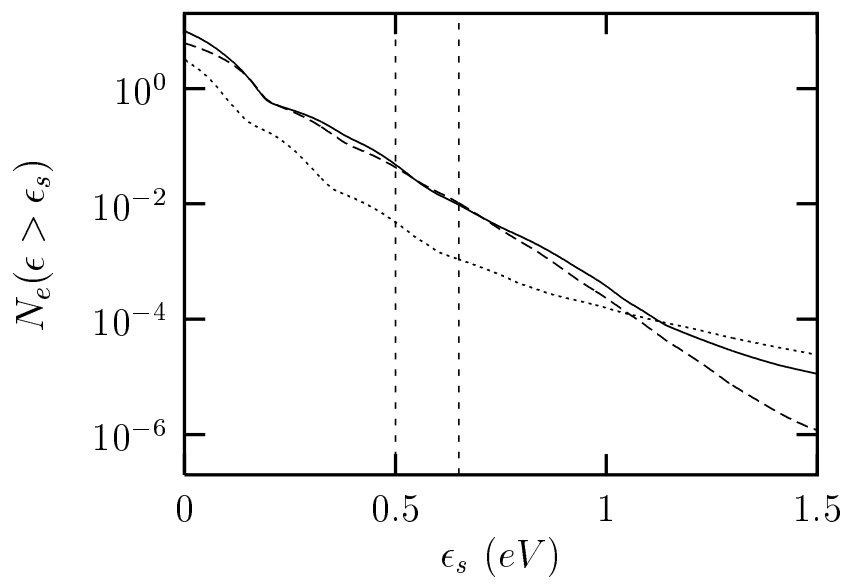

FIG. 9: Number of electrons per atom made available for detection over a Schottky barrier of height $\epsilon_{s}$ for $\mathrm{H}$ incident on the top site of $\mathrm{Cu}(111)$. The vertical lines span the range of Schottky barrier heights found by Nienhaus et al. $\stackrel{6}{\text { for both }}$ $\mathrm{Cu}$ and $\mathrm{Ag}$. Solid line is $N_{e}$ due to the full friction coefficient (as in Fig. 3), dashed line that due $\eta^{s m}$, the smooth part of the profile, and the dotted line that due to $\eta^{p k}$, the peaked part of the profile. ducing an 'extra' friction term to the calculation of the trajectory (not the FOM itself) that would reproduce this energy loss rate were no electron-hole pair creation present. Taking an appropriate friction coefficient and introducing the trajectory at $z=4 \AA$ above the surface (see Fig. 3) results in a decrease in the chemicurrent by an approximately constant factor of $\sim 0.8$ for $\mathrm{H}$, and a decrease in the average number of excited electron-hole pairs from 11.4 to 9.5. Although we stress this is a crude estimate it does suggest that phonon production has a small (though not negligible) influence. For heavier adsorbates a stronger effect would be expected.

\section{CONCLUSION}

An $a b$ initio description of the energy transfer from an adsorbate incident on a metal surface to the electrons present in the surface via electron-hole pair creation has been developed. Energy loss from the adsorbate has been described semi-classically, and the excitation of the electron gas described quantum mechanically using a FOM. It has been shown that a large degree of consistency exists between TDDFT, the classical energy loss of the adsorbate and the energy gain of the substrate electrons, in that the average energy changes are equal. We have also shown that the Markov limit used to define a friction coefficient for the classical motion of the adsorbate has a counterpart in a common Fermi energy approximation for the matrix elements used in the implementation of the FOM.

This classical adsorbate/quantum electronic description of the non-adiabatic electron-hole excitation process has been applied to $\mathrm{H}$ (and $\mathrm{D}$ ) incident on $\mathrm{Cu}(111)$, with somewhat surprising results. We find that a singular friction coefficient results from the application of spindependent DFT. The singular behaviour occurs at the transition point (spin-polarised to spin-degenerate) and is due to the strongly non-adiabatic nature of the evolution of the system around this transition. A breakdown of the nearly-adiabatic definition of the friction coefficient results, suggesting that the friction coefficient cannot be defined for systems exhibiting a spin-transition of this kind. The FOM exhibits the same singular behaviour. Further investigation of this effect suggests that the strongly non-adiabatic system can be replaced by a weakly non-adiabatic 'fixed spin' system that provides a good approximation for the trajectories of the incident atoms considered, avoiding the breakdown of both the FOM and the friction description. Our final results indicate that, provided the singularity in the friction coefficient is removed, the region of the trajectory in the vicinity of the spin transition is not of prime importance.

Comparison of the results presented here with the 'chemicurrent' detected by Nienhaus et al. $\underline{6}^{6}$ using Schottky diode devices has been given in a previous Letter 5 . Calculated and experimental chemicurrents agree well, and we have shown that simple dynamics can reproduce 
the large difference between measured chemicurrents for $\mathrm{H}$ and $\mathrm{D}$. The calculations presented here can be extended in several ways. For an $\mathrm{H}$ atom adsorbate it would be interesting to investigate other sites on the surface and also to consider motion parallel to the surface. Other atomic adsorbates and surfaces can obviously also be analysed, although for heavier species it will be important to treat phonon as well as electron-hole pair excitation. It would also be interesting to consider molecular adsorbates. One question that immediately arises here is whether vibrational or rotational motion is as effective as translation in producing electron-hole pairs.

\section{Acknowledgments}

We acknowledge the support of the UK Engineering and Physical Sciences Research Council (EPSRC) and M.P. is grateful for support from the Swedish Research Council (VR) and the Swedish foundation for strategic research (SSF) through the materials consortium 'ATOMICS'.

\section{APPENDIX}

In this appendix we outline the derivation of the results in Eqs. (1) and (18). To ease the notation we take $\hbar=1$. We begin by deriving the general expression for the non-adiabatic energy transfer in Eq. (1), which is just a variant of the Hellmann-Feynmann theorem. The instantaneous expectation value $E(t)$ of the many-electron system in the presence of the adsorbate is defined as

$$
E(t)=\left\langle\psi(t)\left|\hat{H}_{0}+\hat{V}_{\text {ext }}(t)\right| \psi(t)\right\rangle
$$

where $\hat{H}_{0}$ is the many-electron Hamiltonian, $\hat{V}_{\text {ext }}(t)$ is the time-dependent interaction of the electrons with the moving nucleus, and $|\psi(t)\rangle$ is the many-electron state satisfying the time-dependent Schrödinger equation with the Hamiltonian $\hat{H}(t)=\hat{H}_{0}+\hat{V}_{\text {ext }}(t)$. Using the Schrödinger equation in the evaluation of the time-derivative of $E(t)$ one obtains directly

$$
\begin{aligned}
\dot{E}(t) & =-i\langle\psi(t)|\hat{H}(t) \hat{H}(t)| \psi(t)\rangle+i\langle\psi(t)|\hat{H}(t) \hat{H}(t)| \psi(t)\rangle+\left\langle\psi(t)\left|\frac{\hat{H}(t)}{d t}\right| \psi(t)\right\rangle \\
& =\left\langle\psi(t)\left|\frac{\hat{V}_{\text {ext }}(t)}{d t}\right| \psi(t)\right\rangle .
\end{aligned}
$$

To obtain the non-adiabatic part of $\dot{E}(t)$ we have to subtract the adiabatic part $\dot{E}_{0}(t)$. The adiabatic energy $E_{0}(t)$ is defined in an analogous manner to $E(t)$ in Eq. (A.1) but with the difference that $|\psi(t)\rangle=\left|\psi_{0}(t)\right\rangle$ where $\left|\psi_{0}(t)\right\rangle$ is the instantaneous ground state of $\hat{H}(t)$, that is, $\hat{H}(t)\left|\psi_{0}(t)\right\rangle=E_{0}(t)\left|\psi_{0}(t)\right\rangle$. Using this definition of $\left|\psi_{0}(t)\right\rangle$, a straightforward differentiation gives

$$
\begin{aligned}
\dot{E}_{0}(t) & =\left\langle\dot{\psi}_{0}(t)\left|E_{0}(t)\right| \psi_{0}(t)\right\rangle+\left\langle\psi_{0}(t)\left|E_{0}(t)\right| \dot{\psi}_{0}(t)\right\rangle+\left\langle\psi_{0}(t)\left|\frac{\hat{H}(t)}{d t}\right| \psi_{0}(t)\right\rangle \\
& =\left\langle\psi_{0}(t)\left|\frac{\hat{V}_{\text {ext }}(t)}{d t}\right| \psi_{0}(t)\right\rangle .
\end{aligned}
$$

In the last step we have used the fact that the derivative of the norm of $\left|\psi_{0}(t)\right\rangle$ is zero, that is, $\frac{d}{d t}\left\langle\psi_{0}(t) \mid \psi_{0}(t)\right\rangle=$ $\left\langle\dot{\psi}_{0}(t) \mid \psi_{0}(t)\right\rangle+\left\langle\psi_{0}(t) \mid \dot{\psi}_{0}(t)\right\rangle=0$. Eq. (1) is now directly proved by using the explicit result for $\hat{V}_{\text {ext }}(t)=$ $\int d \mathbf{r} V_{\text {ext }}(\mathbf{r}, z(t)) \hat{n}(\mathbf{r})$ where $V_{\text {ext }}(\mathbf{r}, z)$ is the bare electronnucleus interaction potential and $\hat{n}(\mathbf{r})$ is the electron density operator. Note that $n(\mathbf{r}, t) \equiv\langle\psi(t)|\hat{n}(\mathbf{r})| \psi(t)\rangle$ and $n_{0}(\mathbf{r}, t) \equiv\left\langle\psi_{0}(t)|\hat{n}(\mathbf{r})| \psi_{0}(t)\right\rangle$.

We now turn to the derivation of the expression for the non-adiabatic energy transfer in Eq. (18). Starting with $P_{t}(\omega)$, the probability of the incident atom having lost energy $\omega$ by electron-hole excitation at time $t$, the expectation value of the energy loss is given by

$$
E_{t}=\int_{-\infty}^{\infty} \omega P_{t}(\omega) d \omega
$$

where

$$
P_{t}(\omega)=\frac{1}{2 \pi} \int_{-\infty}^{\infty} \mathrm{e}^{P_{s}(\tau, t)-\alpha_{0}(t)} \mathrm{e}^{\mathrm{i} \omega \tau} d \tau
$$

and $P_{s}(\omega, t) / \alpha_{0}(t)$ is defined as in Eq. (16), but with the upper limit of the time integral taken as $t$. Physically this quantity is the probability that a single electron-hole excitation event is of energy $\omega$, in the time interval $-\infty$ to $t$. Interpreting the factor $i \omega$ as a derivative operator 
in the time co-ordinate leads to

$$
\begin{aligned}
E_{t} & =\left.i \frac{d}{d \tau} \mathrm{e}^{P_{s}(\tau, t)-\alpha_{0}(t)}\right|_{\tau=0} \\
& =\left.i \frac{d}{d \tau} P_{s}(\tau, t)\right|_{\tau=0} .
\end{aligned}
$$

$P_{s}(\tau, t)$ is then expressed in terms of its Fourier transform in $\tau$, giving

$$
E_{t}=\int_{-\infty}^{\infty} \omega P_{s}(\omega, t) d \omega
$$

* Electronic address: jrt32@cam.ac.uk

${ }^{1}$ G. R. Darling and S. Holloway, Rep. Prog. Phys. 58, 1595 (1995).

2 Z. S. Wang, G. R. Darling, and S. Holloway, Phys. Rev. Lett. 87, 226102 (2001).

3 H. F. Busnengo, W. Dong, P. Sautet, and A. Salin, Phys. Rev. Lett. 87, 127601 (2001).

4 J. R. Trail, M. C. Graham, and D. M. Bird, Comp. Phys. Comm. 137, 163 (2001).

5 J. R. Trail, M. C. Graham, D. M. Bird, M. Persson, and S. Holloway, Phys. Rev. Lett. 88, 166802 (2002).

${ }^{6}$ H. Nienhaus, H. S. Bergh, B. Gergen, A. Majumdar, W. H. Weinberg, and E. W. McFarland, Phys. Rev. Lett. 82, 446 (1999).

7 H. Nienhaus, H. S. Bergh, B. Gergen, A. Majumdar, W. H. Weinberg, and E. W. McFarland, Surf. Sci. 445, 335 (2000).

8 H. Nienhaus, Surf. Sci. Rep. 45, 1 (2002)

9 B. Gergen, H. Nienhaus, W. H. Weinberg, and E. W. McFarland, Science 294, 2521 (2001).

10 J. W. Gadzuk, J. Phys. Chem. B 106, 8265 (2002).

11 E. G. d'Agliano, P. Kumar, W. Schaich, and H. Suhl, Phys. Rev. B 11, 2112 (1975).

12 A. Blandin, A. Nourtier, and D. W. Hone, J. Physique 37, 369 (1976).

13 B. Hellsing and M. Persson, Physica Scripta 29, 360 (1984).

14 M. Head-Gordon and J. C. Tully, J. Chem. Phys. 103, 10137 (1995).

15 M. Plihal and D. C. Langreth, Phys. Rev. B 58, 2191 (1998).
It is also possible to obtain higher moments of $P_{t}(\omega)$ in terms of $P_{s}(\omega, t)$ by the same approach.
16 J. P. Perdew, K. Burke, and M. Ernzerhof, Phys. Rev. Lett. 77, 3865 (1996).

17 N. Troullier and J. L. Martins, Phys. Rev. B 43, 1993 (1991).

18 C. L. A. Lamont, B. N. J. Persson, and G. P. Williams, Chem. Phys. Lett. 243, 429 (1995).

19 D. M. Bird, M. Persson, J. R. Trail, and S. Holloway, Surf. Sci. 566, 761 (2004).

20 In calculations, the spin is fixed by keeping separate Fermi energies for the spin up and spin down electrons.

${ }^{21}$ K. Schönhammer and O. Gunnarsson, in Many Body Phenomena at Surfaces, edited by D. Langreth and H. Suhl (Academic, New York 1984).

22 K. Schönhammer and O. Gunnarsson, Phys. Rev. B 22, 1629 (1980).

${ }^{23}$ K. Schönhammer, Z. Phys. B 45,23 (1981).

24 O. Gunnarsson and K. Schönhammer, Phys. Rev. B 25, 2503 (1982).

25 P. Minnhagen, J. Phys. C: Solid State Phys. 15, 2293 (1982).

26 R. Brako and D. M. Newns, J. Phys. C: Solid State Phys. 14, 3065 (1981).

27 M. Persson and J. Harris, Surf. Sci. 189/190, 557 (1987).

28 M. Persson and J. Harris, Surf. Sci. 187, 67 (1987).

29 R. Kubo, J. Phys. Soc. Japan. 17, 1100 (1962).

30 E. Müller-Hartmann, T. V. Ramakrishan, and G. Toulouse, Phys. Rev. B 3, 1102 (1971).

31 J. Strömquist, L. Bengtsson, M. Persson, and B. Hammer, Surf. Sci. 397, 382 (1998). 